\title{
The Development of a Community-Based Drug Intervention for Filipino Drug Users
}

\author{
Ma. Regina M. Hechanova, ${ }^{1}$ Arsenio S. Alianan, ${ }^{1}$ Mendiola T. Calleja, ${ }^{1}$ Isabel E. Melgar, ${ }^{1}$ \\ Avegale Acosta, ${ }^{1}$ Angelique Villasanta, ${ }^{1}$ Kay Bunagan, ${ }^{1}$ Camille Yusay, ${ }^{1}$ Angelica Ang, ${ }^{1}$ \\ Jane Flores, ${ }^{1}$ Nico Canoy, ${ }^{1}$ Ervina Espina, ${ }^{2}$ Gayle A. Gomez, ${ }^{3}$ Elena Samonte Hinckley, ${ }^{4}$ \\ Antover P. Tuliao, ${ }^{5}$ and Miriam P. Cue ${ }^{6}$ \\ ${ }^{1}$ Ateneo de Manila University, Loyola Heights, Quezon City, Philippines \\ 2 University of the Philippines - Visayas Tacloban, Tacloban City, Leyte, Philippines \\ 3 Ateneo de Naga University, Naga, Camarines Sur, Philippines \\ ${ }^{4}$ University of the Philippines - Diliman, Quezon City, Philippines \\ ${ }^{5}$ Community, Family, and Addiction Sciences Department, Texas Tech University, Lubbock, Texas, USA \\ 6 Philippine Regulatory Commission, New Day Recovery Center, Davao City, Davao Del Sur, Philippines
}

\begin{abstract}
T his article documents the development of a community-based drug intervention for low- to mild-risk drug users who surrendered as part of the Philippine government's anti-drug campaign. It highlights the importance of developing evidence-informed drug recovery interventions that are appropriate to the Asian culture and to developing economies. Interviews and consultations with users and community stakeholders reveal the need for an intervention that would improve the drug recovery skills and life skills of users. Evidence-based interventions were adapted using McKleroy and colleagues' (2006) Map of Adaptation Process (MAP) framework. The resulting intervention reflected the country's collectivist culture, relational values, propensity for indirect and non-verbal communication, and interdependent self-construal. The use of small groups, interactive and creative methodologies, and the incorporation of music and prayer also recognised the importance of these in the Philippine culture.
\end{abstract}

Keywords: drug use, addiction, community-based drug treatment, Philippines, program adaptation

The past decades have seen increasing concern for substance use, and the United Nations Office of Drugs and Crime (UNODC) reports that $2.3 \%$ of the world's population abuses illicit substances (UNODC, 2014). The Philippine Drug Enforcement Agency (PDEA) also reported that $92 \%$ of barangays ${ }^{1}$ in the National Capital Region are affected by illicit drug use (PDEA, 2015). In July 2016, newly elected President of the Philippines Rodrigo Duterte declared a 'war' against illicit drugs. Dubbed 'Operation Double Barrel' or Oplan Tokhang, ${ }^{2}$ the campaign involved efforts at demand reduction and supply reduction. Supply reduction involved shutting down drug laboratories and arresting drug suppliers. Demand reduction activities involved community officials and/or local police going to the homes of known users and asking them to voluntarily surrender and receive treatment. As of March 2017, 1.18 million illicit drug users have surrendered (Raymundo, 2017) with an additional 44,070 arrests and 2,206 fatalities (Palatino, 2017). However, beyond these legal measures, the Human Rights Watch (2017) reports that the Philippine National Police and unidentified vigilantes have killed about 7,000 suspected drug users and pushers in what are now termed extra-judicial killings.

The staggering numbers highlight the urgent need to develop and implement drug treatment programs. However, because drug treatments are costly and generally inaccessible to the poor, the UNODC (2014) suggested the necessary provision of community-based drug recovery support. This involves providing holistic care in the form of preventive education, health promotion, screening, treatment and rehabilitation services, primary health services, basic support, education, skills training, and livelihood opportunities in communities (UNODC, 2014). Beyond being cost-effective, there is evidence that community-based drug interventions have significantly decreased hospital stay, emergency room visits, and criminality (UNODC, 2014). 
The Dangerous Drugs Board (2016) of the Philippines reported that $90 \%$ of those who have voluntarily surrendered could be treated in the community (Cepeda, 2016). However, like most countries in Asia, the Philippines has primarily employed compulsory residential treatment in resolving illicit drug use problems (Vuong et al., 2017). Given the overwhelming number of clients, communities have created their own programs consisting of community service (e.g., cleaning, beautification, tree planting, gardening), recreational activities (e.g., sports activities, Zumba, yoga), counselling, and spiritual formation (e.g., bible study, prayer groups).

International guidelines advocate the use of evidenceinformed and culturally nuanced drug treatments (UNODC, 2014). Although there is literature on evidencebased community interventions, they have emanated from developed and Western countries, and there is a dearth of literature from low- to middle-income countries (LMIC) in Asia. This study sought to fill the gap by describing the adaptation and design of a community-based drug recovery program for low- to mild-risk Filipino users. It further adds to the literature by highlighting the psychosocial needs of Filipino users and cultural and contextual considerations, thus supporting models of program adaptation that incorporate stakeholder and cultural factors.

\section{Community-Based Programs}

The UNODC (2014) describes illicit drug use as a complex health condition that has social, psychological, and biological dimensions. Rather than addresssing illicit drug use from a criminal justice perspective, UNODC advocates that it should be treated as a health condition with community-based treatment offered as an alternative to incarceration when possible. In contrast to centre-based residential rehabilitation, community-based treatment is primarily outpatient rehabilitation. Community-based treatment programs ascribe to the following key principles: minimal disruption to stakeholder support systems, comprehensive continuum of care, evidence-based practices, acceptance of program implementers, and culturally appropriate (UNODC, 2014). This approach acknowledges the complexity of drug use and involves the client's family and the community to ensure efficient and long-term results. The UNODC advocates a holistic approach that includes needs assessment, treatment planning, program implementation, and case management. Another key principle in community-based treatment is the provision of a continuum of care that takes a comprehensive approach in addressing the stakeholders' general health, family, education, and employment needs. It also highlights the active role of people affected by drug use and their families and community members in the service planning and delivery (UNODC, 2014).
UNODC also recommends the use of evidence-based treatments, and there is growing literature on outpatient treatments for illicit drug use and misuse (McCarty et al., 2014). The most robust evidence has been reported on cognitive behaviour therapy (CBT; Windsor, Jemal, \& Alessi, 2015). CBT assumes that drug use is a learned behaviour and thus can be unlearned through therapeutic strategies (Magill \& Ray, 2009). It focuses on changing behaviours by addressing maladaptive cognitions and emotions that shape behaviour (Beck, 1970). Another popular approach that has been shown to produce small but sustained reduction in substance use is motivational interviewing (MI; Sayegh, Huey, Zara, \& Jhaveri, 2017). MI assumes that people will not change simply because they are told to do so and that real change needs to come from within. It is a client-focused approach that aims to elicit internal motivation to change through the experience of empathy, increasing an internal sense of discrepancy between ones's goals and values and one's current situation, and fostering a sense of self-efficacy and optimism (Miller \& Rollnick, 2012). The community reinforcement approach (CRA) involves the use of rewards to spur positive behavioural changes (Roozen et al., 2004). Founded on operant conditioning and behavioural modification principles, clients are encouraged to set goals and are given rewards for achieving sobreity-related behaviours and goals. It has been found effective for people with alcohol use problems and has been adapted for illict drug use (Meyers, Roozen, \& Smith, 2011). Finally, another program that has evidence of effectiveness is the 12-Step Program (Ouimette, Finney, \& Moos, 1997). The 12-Step Program is founded on a belief of a 'power greater than oneself' among recovering users. Its principles include accepting addiction as a disease, embracing sobriety, surrendering to a higher power, self-reflection and assessment, seeking reparation, and deepening one's faith. It highlights the importance of mutual support though sponsors and participation in 12-Step community sessions (Baker, Daley, Donovan, \& Floyd, 2009).

Given the many alternatives, a critical factor in selecting community-based strategies is the acceptance of those who will implement them. A study among community mental health staff reports that they are more inclined to use interventions that are flexible and easy to implement (Nelson, Steele, \& Mize, 2006). Resistance to MI was mostly due to perceived difficulty and the lack of training. Challenges in the use of CBT include perceived low motivation and cognitive ability of patients, and psychiatric instability of some clients. Barriers to using CRA include difficulty in certification requirements, resistance from clients, and the lack of resources to implement (Amodeo, Cohen, Chassler, \& D'ippolito, 2011). The challenges cited for the12-Step Program were resistance from clients, non-participation and attendance, possibility of one client dominating the discussion, and the need of clients for answers or solutions to their problems (Baker et al., 2009). 
Considerations in Implementing Community-Based Interventions in the Philippines

Beyond utilising evidence-based interventions, international guidelines advocate the adaptation of evidencebased treatment methodologies to local context and culture (UNODC, 2014). Cultural adaptation requires understanding local culture and context. A major challenge in implementing community-based drug treatment in the Philippines is the lack of resources allocated for mental health services. The country's budget for health is only $2-3 \%$ of the national budget, and mental health is a low priority. There is also a dearth of mental health professionals, as well as a disparity in their distribution. For example, there is only one licensed psychologist for every 100,000 Filipinos, and most psychiatrists and psychologists are located in urban areas (Hechanova et al., 2015).

Another possible barrier to community-based interventions is the prevailing stigma on drug users and a reluctance to seek treatment (Hechanova, Tuliao, Teh, Alianan, \& Acosta, 2013; Tuliao, Velasquez, Bello, \& Pinson, 2016). Studies report that Filipinos are generally reluctant to open up to strangers because they believe that seeing a professional means they are crazy, and this will tarnish the reputation of their family (Hechanova et al., 2013). Rather than professionals, Filipinos prefer to seek help from family, friends, religious leaders, and folk healers (e.g., Abe-Kim, Gong, \& Takeuchi, 2004; Tuliao, 2014).

The Philippines has also been described as a collectivist and interdependent culture. Filipinos have a difficult time saying 'no' to friends and family (Hechanova \& Waelde, 2017; Tuliao, 2014). On the one hand, the tendency to conform can be a challenge for drug users who want to quit. On the other hand, this can be harnessed by incorporating reference groups into the intervention strategy (Dueck \& Byron, 2012). This is supported by evidence that groupbased interventions are a good venue for healing among Filipino survivors (Hechanova et al., 2015; Hechanova, Waelde, \& Ramos, 2016).

The Philippine culture is strongly rooted in spiritual and religious traditions. Filipinos draw inner strength and support from their faith, and this functions as a protective factor, especially among survivors of natural disasters (Hechanova et al., 2015; Hechanova \& Waelde, 2017). Participation in church rituals and prayer groups is a means to cope, and religious communities are important social capital (Nakonz \& Shik, 2009). As such, community-based interventions may harness psycho-spiritual resources to enable healing.

Finally, another consideration in designing psychological interventions in the Philippines is emotional expression. The Philippine culture has roots in Chinese culture, and a common belief among Chinese is that talking about painful issues and experiences causes excessive and unbalanced emotions that lead to illness (Haque, 2010). Dueck and Byron (2012) suggest that in such cultures, projective approaches, such as using art and theatre, can facilitate emotional expression. Studies show that disaster interventions in the Philippines incorporate music (e.g., Hechanova et al., 2015) and other art forms (e.g., Parr, 2015).

\section{Research Problem}

Given that cultural nuances are important in the cultural adaptation and design of community-based interventions, this article documents the development of a communitybased drug recovery intervention for low- to mild-risk illicit drug users in the Philippines. This study used McKleroy and colleagues' (2006) Map of Adaptation Process (MAP) framework, which prescribes five phases: (1) assessment of the needs, risk factors, and implementation considerations; (2) developing the intervention model and determining adaptations; (3) training facilitators and pretesting the materials; (4) pilot-testing; and (5) implementation of the adapted intervention. This article describes the first three phases of the aforementioned process and sought to answer the following research questions:

1. What are the psychosocial needs and risk factors of Filipino drug users?

2. What cultural factors need to be considered in the design and adaptation of the modules?

3. What are the factors that need to be considered in the delivery of the modules?

\section{Methods}

The study utilised a mixed-method design using multiple data sources such as interviews with drug users and focused group discussions with community stakeholders.

\section{Needs Analysis Interviews}

In-depth interviews were conducted in Metro Manila among 48 illicit drug users who had voluntarily surrendered and consented to participate in the interviews. The majority of participants were male $(n=35,72 \%)$, married or co-habiting $(n=30,64 \%)$, with an average age of 36 years (range $18-50$ years). About half ( $n=25,52 \%)$ were employed in manual and contractual work (e.g., construction work, electrician, street sweeper). The majority reported they had used methamphetamine $(n=34,70 \%)$, followed by marijuana $(n=10,20 \%)$ and inhalants $(n=$ $5,10 \%)$.

The interview questions included the following: (1) How did you start taking illicit drugs? (2) Have you tried to stop using illicit drugs? What happened? (3) What were you feeling, thinking, or doing the last time you used illicit drugs? (4) What are the benefits you get from using illicit drugs? (5) What are the negative effects of illicit drugs on you? (6) What do you do when you feel the craving to use? (7) What or who made you want to stop using illicit drugs? (8)What are your dreams in life? What are your plans to achieve these dreams? (9) Have you tried asking for help to stop using illicit drugs? Why or why not? (10) What help would you need to stop using illicit drugs? 
Drug dependence. The World Health Organization (WHO) ICD-10 Symptom Checklist was verbally administered to measure drug dependence symptoms (WHO, 2004). The items include cravings, control of use, withdrawal symptoms, pattern of use, neglect of other interests in favour of drug use, experience of physical or psychological harm, and persistence of use despite consequences. Drug dependence is indicated if three or more symptoms are present.

\section{Consultations With Community Stakeholders}

Two consultation discussions were conducted with community stakeholders. The first consultation aimed to obtain contextual and cultural considerations to aid in the design adaptation of the drug-recovery intervention. The second consultation aimed to validate the design of the intervention and elicit considerations in the delivery of the intervention.

The first consultation was conducted with 15 mental health professionals $(39 \%)$, three addiction counsellors $(8 \%)$, and 20 community stakeholders, including recovering users (52\%). Individuals were invited based on their exposure to drug use and involvement in existing community-based drug rehabilitation. Stakeholders were divided into smaller groups, assigned a component of the model, and tasked to examine the Matrix Intensive Outpatient Program (MIOP) of the Substance Abuse Mental Health Services Administration (SAMHSA, 2006) and the UNODC (2015) Trainer's Manual: Community-Based Services for People Who Use Illicit Drugs in Southeast Asia. Groups were asked to examine the draft modules based on three facets: (1) deep structure, (2) surface structure, and (3) delivery. Participants were asked the following questions: (1) To what extent is the core theory of change consistent with the characteristics, beliefs and norms of the target population? (deep structure); (2) To what extent does the methodology, message and materials match the target population? (3) What other factors should be considered in the design of the intervention? (surface structure).

The second consultation aimed to validate the design and obtain inputs from stakeholders on program implementation and delivery. Participants from the previous design meeting were invited, but only 29 were able to attend. The group was composed of $59 \%$ community stakeholders $(n=17), 32 \%$ mental health professionals $(n=9)$, and $10 \%$ addiction professionals $(n=3)$. The draft modules were presented and community stakeholders were asked the following questions: (1) To what extent does the methodology, message and materials match the target population? (2)What factors need to be considered in the delivery of the intervention?

\section{Procedure}

Ethics approval was obtained from the Ateneo de Manila University Institutional Review Board. Informed consent was obtained from all participants in this study. Participants were assured that all information would only be for research purposes and would not be revealed to community officials. To protect the privacy and confidentiality of interviewees, interviewers asked them not to use their real names or cite real names of other people. The data were accessible only to researchers who were not members of the community. The data from interviews and consultations were thematically analysed. After an initial read through of the data, themes were identified, and another researcher was assigned to perform a frequency analysis of these themes.

\section{Results}

\section{Profile of Illicit Drug Users}

Severity of drug dependence. Based on the prescribed cutoff scores on the ICD-10 scale of psychoactive substance use (WHO, 2004), results showed only $14 \%$ of participants had scores indicative of full dependency, $39 \%$ had some symptoms, and $47 \%$ had no symptoms of drug dependence. The most frequently identified dependence symptoms were compulsions or cravings, neglecting responsibilities and interests, and continued use despite harmful consequences.

Chronic nature of drug use. The results of the interviews confirmed the chronic nature of drug use. Half of participants (50\%) had attempted to quit. Of these, $18 \%$ had lapsed once, $41 \%$ had lapsed twice, $12 \%$ had lapsed thrice, $12 \%$ had lapsed $4-6$ times, and $17 \%$ lapsed more than this.

Reasons for drug use. The interviews indicated four factors influencing use: peers, family, work, and personal. The most common reason given for use and lapse was peer influence ('My friends invited me'), family problems ('My father beats me', 'My wife and I separated', 'Fights at home') and family influence ('My uncle was using'). Others cited work reasons ('Gives me more energy', 'I can work better'). Individual factors included the desire to experiment ('I just wanted to taste'), mood ('Wanted to feel good'), and boredom ('Didn't have work, nothing to do').

Benefits of drug use. The most commonly cited benefit $(45 \%)$ of drug use was increased energy and productivity (e.g., 'Lets me stay awake,' 'Feel less tired, more energised'). Participants described a tamang sipag (energy trip) that allows them to work longer and harder. The second most common benefit mentioned (23\%) was positive mood ('I felt happier').

Negative effects of drug use. A number (43\%) of participants did not report any negative effects from using illicit drugs. However, those with dependence symptoms reported negative effects on their health (e.g., stroke, stomach aches, dry skin), relationships (e.g., separation of family, marital conflicts), and mood (e.g., bad temper, irritability). Other negative impacts included illegal activities (theft), financial losses ('Money for family went to illicit drugs'), tamang hinala (paranoia) and mental health issues (hallucinations). 
Help-seeking. The majority of participants (55\%) said they had never sought help for their drug use because they felt they did not need help ('I thought I could stop on my own') or felt that drug use was not a problem ('I can stop any time'). Those who had sought help had approached their family (76\%), a church (14\%), or community member $(14 \%)$. However, there were also those who did not know who to approach ('I didn't know who to ask') or were ashamed to seek help ('I was ashamed').

Coping strategies. Non-dependent users reported adaptive coping strategies such as avoidance ('I stay at home'), looking for distraction, keeping busy, exercise, thinking about impact on family, praying, sleeping, and eating. However, those with dependence symptoms reported non-adaptive behaviours such as resorting to drug use ('I take again'), coercing family members for money to buy illicit drugs ('I make my wife give me money to buy illicit drugs'), and even stealing or pushing to obtain the funds to buy illicit drugs.

Motivation to change. The majority (60\%) of participants stated their motivation to stop was their family. They reported pressure from their families to stop, as well as a desire to provide a better life for their families. Sixteen percent cited community officials and the fact that they were personally approached. Fourteen percent said they wanted to quit for themselves and cited personal goals (i.e., desire to have steady work, to continue studies, achieve career goals and improve their health). However, they also were not clear on how to go about achieving these. About $9 \%$ cited the government's drug war and the fear of being incarcerated or killed as the reason they surrendered.

Support needed. When asked what support they needed, $57 \%$ of the participants cited job opportunities and $56 \%$ cited emotional support from family. About a third (36\%) cited community programs for recovering users and $15 \%$ verbalised their hope that their communities could keep them safe and protect them from pushers and vigilantes.

\section{Considerations in the Design of the Intervention}

Consistency of core theory of change with the target population. Stakeholders affirmed the importance of the interventions. As one participant said: 'People say "Just say no". If it is that easy, there would be no drug addicts. The question is, how do you say "no"?' They observed that current initiatives such as community service, prayer sessions, and exercise programs were helpful diversions but not adequate ('Zumba and prayer can only do so much'). Stakeholders reported attrition and a return to illicit drug use among those in community diversion programs. As explained by one community member: 'The motivation to quit is there, but that's not enough if they don't know how.' This validated the importance of focusing on changing motivations, behaviours, cognitions, and emotions to help drug users stay sober.

Literacy and learning style. Stakeholders affirmed the relevance of the MIOP and UNODC modules but raised con- cerns about their suitability given participants' low literacy levels. Stakeholders suggested the need to simplify the modules and use fewer cognitive approaches: 'Our participants can't read or write, so worksheets and reading materials won't work.' This validated the data obtained that clients were predominantly under-educated. Community stakeholders also suggested using videos, visuals, physical activities, and creative methodologies such as storytelling, music, and art to engage participants.

Resources for implementation. The stakeholder consultation also highlighted the lack of preparedness and resources of local government units to implement community-based drug recovery support. Participants reported the lack of budget, facilities, manpower, and resources to implement these programs and, given this, suggested the need to minimise required materials and equipment.

Religious beliefs. Finally, stakeholders suggested the importance of acknowledging participants' religious beliefs in the design of modules. Although some suggested the inclusion of Bible verses, others felt that this would exclude Muslim and non-Christian participants. Addiction professionals suggested the use of ecumenical prayers such as the Serenity prayer (popularised by the 12-Step Program), given the possible diversity of participants.

\section{Design and Adaptation of the Intervention}

Based on the needs analysis and stakeholders' inputs, 12 modules were designed for low- to mild-risk users. The first six modules focused on drug recovery skills and were based on the Brief Assist Intervention by the WHO (2010), the MIOP (SAMHSA, 2006), and the UNODC's (2015) Manual on Community-Based Services. The next six modules, which were focused on developing life skills, were adapted from SAMHSA's (2006) MIOP and Katatagan, a resilience program developed for Filipino disaster survivors (Hechanova et al., 2015).

The modules were founded on principles of MI that focus on eliciting internal motivation and enhancing selfefficacy. CBT principles were used to shape ineffective thoughts, emotions, and behaviours. Mindfulness was also incorporated in each module to centre participants and build their capacity for self-awareness and self-regulation. Given the collectivist culture, the intervention was designed to be delivered in the form of structured group sessions. Each module consisted of specific parts: welcome, mindfulness-centring exercise, review of previous session and homework, module proper, reflection on insights, homework, and closing. Modules were designed using principles of adult learning; namely, salience (based on needs), practice, application and reinforcement (exercises, role-playing, application, and homework). Recognising the value of the arts in Philippine culture, drawing activities are used when appropriate, and sessions end with a prayer or a song. Even though the Serenity prayer was suggested, the manual emphasised the flexibility of 
facilitators in choosing appropriate prayers or songs depending on the religious beliefs of their participants.

The introductory module (Understanding Drug Addiction), aims to educate the participants about the effects of illicit drugs and the nature of addiction. The session begins with a local video on the experiences of Filipino drug users that serves as a springboard to elicit participants' own experiences. This is followed by a discussion on stages of drug use, the impact of illicit drugs, and the myths about drug use. An overview of the entire program is presented and participants are invited to attend the program. Because studies show that groups enable healing in interdependent and collectivist cultures (Hechanova et al., 2015; Hechanova et al., 2016), participants are asked to come up with a group name in order to build social support and a sense of group identity. Recognising the interdependent nature of Filipinos, the homework for this session is to ask family and friends how the participants' drug use had affected them.

Module 2 (Importance of Change) is based on MI theory that proposes that real change happens when it comes from within (Miller \& Rollnick, 2012). The highlight of the module is the processing of the discrepancy between participants' actual and ideal life and identifying their motivations for changing. Participants are also asked to reflect on the importance and their readiness and self-confidence in remaining sober. Recognising the interdependence on and central role of family and friends for Filipinos (Enriquez, 1978), the homework for this module is for participants to ask their family and/or friends how important is it for them to change.

Module 3 (Coping with Cravings) was designed to respond to the finding that users lacked adaptive coping skills to remain sober. The module recognises that cravings are an inherent part of recovery and focuses on strategies to manage cravings (e.g., delay, distract, decide; thought stopping and thought substitution). Given the low literacy level of participants, these concepts are taught using interactive methodologies such as structured learning experience, drawing of symbols, and the creation of a slogan.

Most interviewees cited external triggers as reasons for use. Given this, Module 4 (Managing External Triggers) begins with a structured learning experience where participants are asked to go through an obstacle course while blindfolded. They are then asked to reflect on the role of one's environment and the importance of being aware of the external factors - people, places, things or events that trigger their use. Cognisant of Filipinos' concern to protect family members and friends from shame (Hennig, 1983; Lynch, 1962), participants are asked to use pseudonyms.

The interviews highlighted the influence of peers on drug use. Module 5, entitled 'Saying No', aims to develop drug refusal skills. These skills are particularly important given the country's interdependent culture and the fact that Filipinos have a difficult time saying 'no' to friends and family (Hechanova \& Waelde, 2017; Tuliao, 2014). In the first part of the module, participants are asked to rate friends and relatives on the extent of their influence on their drug use. This is followed by a discussion on ways they can avoid interactions with these people. The second part is spent role-playing different ways of refusing offers of illicit drug use

The goal of drug recovery does not only include abstinence but also the achievement of improved health and quality of life. Module 6 (Adopting a Healthy Lifestyle) seeks to address the negative effects of drug use by equipping participants with various self-care strategies. It uses the Eight Dimensions of Wellness (i.e., social, environmental, occupational, spiritual, material, emotional, intellectual, and physical), which was chosen because of its inclusion of spirituality (SAMHSA, 2016). Faith is often cited in local literature as an important means of support for Filipinos (Hechanova \& Waelde, 2017; LadridoIgnacio, 2011). They see hardships as spiritual opportunities, prayer as a means of coping, and religious communities as an important social resource (Nakonz \& Shih, 2009).

The needs analysis revealed that negative emotional states are triggers for use. Module 7 (Managing Thoughts and Emotions) aims to help participants manage negative emotions through the use of CBT principles, specifically Ellis's (2001) ABC-DEF Model of Emotional Disturbance. However, a study among Filipino disaster survivors revealed that participants have difficulty parsing thoughts from feelings (Hechanova et al., 2015). To aid participants, the module begins with a vignette to highlight the relationship between emotions, thoughts, and behaviours. Participants are then shown ambiguous pictures, leading to a discussion on interpreting the same precept in different ways. This is followed discussion and exercises on disputing irrational beliefs and developing alternative thoughts that can lead to new emotions and behaviours. An acronym was created using Filipino terms to enable better recall of this reframing process.

The interviews revealed that drug use led to aggressive behaviour, relationship problems, and isolation. The literature highlights the Filipino value of kapwa ('unity of the self with others'; Enriquez, 1992) and the importance of maintaining good relations (Lynch, 1962). Module 8 (Relating to Others) aims to improve participants' relationships with families and friends by developing interpersonal skills. Because communication is a key ingredient in successful relationships (e.g., Nicotera, 1993), this module focuses on communication styles and the importance of active listening. Because of Filipinos' tendency to rely on non-verbal behaviour and pakikiramdam, or relational sensitivity to others (Reyes, 2015), the module includes an exercise on sensitivity to non-verbal behaviours.

The interviews revealed the existence of broken relationships because of drug use. Recognising the central role of family and friends in a collectivist culture (Enriquez, 1978), Module 9 (Rebuilding Relationships) aims to begin the process of rebuilding connections by enabling participants to reflect on how they may have been hurt as well 
as hurt others. Participants are invited to contemplate on their readiness to forgive others as well as ask for forgiveness from others. The module ends with a self-compassion exercise encouraging participants to move towards forgiving the self, a major component of compassion-focused therapy (Gilbert, 2014). As part of their homework, participants are asked to make amends with someone they may have hurt in the past.

Recovering users shared multiple problems such as poverty, lack of stable work, conflicts with family, separations, lack of education, and so on. Given these concerns, Module 10 (Problem Solving) focuses on problemsolving skills. It begins with a vignette, and participants reflect on a chain diagram of interrelated problems and identify what might be the root problem. Participants are asked to identify a current problem, brainstorm possible solutions, identify barriers to solving the problem, decide on the most appropriate solution, and plan how to implement it. A cultural nuance in this module involves asking participants whether the problem they cited is within their control. This is because a study reveals that Filipinos tend to take on other people's problems as their own (Hechanova et al., 2015). Based on evidence that for Filipinos, extended family and community members are a critical source of support (Hechanova et al., 2015), participants are also asked to identify possible sources of support.

Interviewees cited experiencing hiya (shame) and loss of face because of the stigma of illicit drug use. Given this, Module 11 (Recognising My Strengths) focuses on building participants' self-esteem and self-efficacy. The module asks participants to identify their strengths as well as their areas of improvement. Given the aforementioned interdependent culture, participants are invited to affirm each other to strengthen participants' self-efficacy and selfesteem. As part of their homework, participants are asked to also obtain feedback from family and friends.

In the final module (Meaning Making and Looking to the Future), participants are invited to reflect on the meaning of their experiences of illicit drug use and what they have learned from it. Given the results of the needs analysis that most recovering users had aspirations but no concrete plans on how to achieve their goals, participants are led through a visioning and planning exercise. The interviews also revealed that participants' motivations mostly revolve around their family. This is consistent with findings that, rather than self-actualisation, what is important for Filipinos is the actualisation of their family members (Ilagan, Hechanova, Co, \& Pleyto, 2014). Thus, the process of planning for one's future recognises that participants' dreams are not just for themselves but may also revolve around their families.

\section{Implementation Considerations}

The second consultation with community stakeholders and addiction professionals sought to validate the de- sign of the interventions and identify considerations for implementation. Participants affirmed the overall design, objectives, and content of the modules. However, they raised a number of factors relevant to the design.

Language and content. Although community stakeholders appreciated that the modules were written in the national language, they commented that some Filipino terms used were unfamiliar. Some stakeholders were concerned that Filipino is not the native language in Central and Southern Philippines, and some key words mean different things (e.g., libang means 'to distract' in Tagalog-based Filipino but means 'to defecate' in Cebuano). A suggestion was to translate the manual to major dialects. Another recommendation was to simplify the language and use the more colloquial 'Taglish' (mixture of Tagalog and English) when appropriate, and translate modules to major dialects. Stakeholders also suggested simplifying the modules teaching CBT principles (reframing, thought substitution) because these were deemed too complex for the target population.

Screening and assessment. The community-based treatment modules were intended for low- to mild-risk users. However, stakeholders reported that there was a bottleneck because the Philippine law stipulates that only government-accredited doctors are allowed to conduct drug dependency evaluation. Given that there are fewer than 500 of these professionals in the entire country, only a small percentage of recovering users have been screened and assessed. This has led to many not being able to get the help they need in a timely manner.

Capability of community facilitators. A concern raised by stakeholders was the lack of community facilitators to deliver the modules. Although they reported having volunteers, particularly from church-based organisations, the majority of these volunteers lacked the capability to facilitate. Stakeholders noted that current programs tended to use lectures and do not focus on skills development and adult-learning methodologies. They affirmed the need to provide community facilitators with facilitation skills and to include simulation of the modules. Given the lack of mental health professionals in their community, another concern was the lack of personnel to monitor and coach community facilitators.

Safety and security. Given the presence of drug pushers in the community and the incidence of extra-judicial killings, a concern raised by stakeholders was the safety of participants and facilitators. Community leaders reinforced the importance of working with law enforcers to safeguard the security of participants and facilitators. To mitigate this risk, the designers added a checklist on the readiness of communities, which includes an assessment of safety. The training also emphasises the importance of working with local government units 
who can ensure the security of those involved in the program.

\section{Discussion}

This article describes the development of an evidenceinformed, community-based drug recovery intervention for low- to mild-risk illicit drug users in the Philippines. Using McKleroy and colleagues' (2006) MAP framework, the study highlights the value of action research and employing a systematic process of adapting interventions to make them more suited to a particular culture and context. In particular, the use of participatory research provided valuable inputs in adapting, designing, and implementing the intervention. Although the iterative process required more time, the investment in the participatory research enhanced the relevance of the intervention.

The resulting intervention consisted of drug recovery skills and life skills. Although these skills are not necessarily unique to Filipinos, the design of the modules reflect important cultural and contextual nuances, including the use of small groups, interactive and creative methodologies, and the use of music and prayer when appropriate. The modules also considered Filipino cultural values such as the importance of family and friends, the propensity for indirect communication, the desire not to offend family and friends, and an interdependent self-construal.

Beyond the design of the intervention, the needs analysis suggests the importance of community preparation and priming of potential participants. Based on the Stages of Change Model (Prochaska, DiClemente, \& Norcross, 1992), those who surrendered appeared to be in the precontemplation stage and may not really see the need for change. In addition, some use illicit drugs for productivity and rationalised it as functional. Hence, low- to mild-risk users may need greater motivation to actually participate in recovery intervention. This suggests that those involved in screening and assessment may need to possess basic skills in motivation enhancement.

In addition, the ambivalence of low- and mild-risk users may be exacerbated by the reluctance of Filipinos to seek professional help (Nadal, 2011; Tuliao et al., 2016). One implication of this is the need to tap community healers and leaders who are the preferred sources of help (e.g., Abe-Kim et al., 2004). The literature also suggests that among Filipinos, health and mental health decisions are made with the family (Nadal, 2011). This suggests that engaging and obtaining the support of family members may be important in encouraging the participation of drug users.

The barriers cited by community stakeholders, such as the lack of training, resistance from clients, clients' limited cognitive ability, and the lack of resources are similar to that faced in other developing countries (Amodeo et al., 2011). This highlights the importance of investing in selecting and training of staff members to be more culturally adept and develop effective skills in delivering these inter- ventions. Communities also need to provide resources for food and transportation, and consider offering incentives for completion of the program, similar to CRA (Roozen et al., 2004).

Another concern was regarding the bottleneck in screening of users. Given that in other countries, other health care professionals are allowed to conduct drug dependency assessment (UNODC, 2014) suggests that current law may not have been drafted with a public health perspective. There appears to be a need to revise the law as well as increase the number of those who can do drug dependence evaluation.

The Philippine government's approach in criminalising illicit drug use and using fear as a means to get people to stop is also not unique. Asian countries such as Vietnam, China, Cambodia, Indonesia, Malaysia, and Thailand likewise adhere to the philosophy of social reeducation and force people who use illicit drugs into compulsory rehabilitation (Vuong et al., 2017). However, the rise in extra-judicial killings and reports that police receive cash rewards for executing drug suspects (Mogato \& Baldwin, 2017) is a critical factor that may affect the success of community-based programs. Some participants and prospective facilitators expressed fear for their lives. If community interventions are to succeed, it is important to ensure the safety of recovering users. The issue of security is just as salient among volunteer community facilitators who may think twice about being involved in working with illicit drug users out of fear for their own safety.

As suggested by UNODC (2014), drug use is a complex phenomenon, and there is a need for a holistic perspective on the issue of drug use. A study of community-based drug recovery in China reports that a lack of coordination, divergent attitudes, and conflicting targets for police and health officials undermine the shared goal of treatment (Ma et al., 2016). This appears to true in the Philippines as well. The lack of recognition of the national government on the many factors that lead to drug use (i.e., poverty, lack of education, unemployment, poor parenting, poor coping skills) suggests the need to educate government leaders and law enforcement officials on the bio-psychosocial aspects of illicit drug use and the need for holistic, long-term and evidence-based solutions.

\section{Limitations of Research and Implications for Future Research}

This study describes the development of an evidenceinformed, community-based drug treatment program. However, there is still a need for robust evaluation of the effectiveness of the intervention. In addition, future studies may wish to explore what factors may influence the effectiveness of the intervention, such as the background of the facilitator, modality of module delivery, group constitution and size, among others.

The intervention was developed for low- to mild-risk adult users in the Philippines. The needs analysis was also conducted only among urban poor drug users. Future 
researchers may wish to validate whether the psychosocial needs of drug users in rural areas and of other socioeconomic classes are different. In addition, community stakeholders also articulated the need for modules for children and youth.

Finally, the study focuses on addressing the psychosocial needs of illicit users. There is emerging literature on the value of recovery support resources such as the role of family and friends. Future studies may wish to examine how these, along with other community resources, may influence the recovery of drug users.

Limitations notwithstanding, the study presents the process of adapting and designing an evidence-informed community-based drug treatment intervention. It highlights the importance of action-research as well as a participative process with stakeholders in the development of interventions for the community.

\section{Acknowledgments}

The authors wish to acknowledge the Psychological Association of the Philippines, Quezon City and the QC Anti-drug abuse council, the Archdiocese of Novaliches, Novaliches Barangay Proper, Commission on Higher Education, United Nations Office of Drug and Crime Philippine Office and all those who were involved in the needs analysis and stakeholders consultations.

\section{Endnotes}

1 Barangay is the smallest unit of governance in the Phillipines.

2 'Tokhang' is a Visayan word contraction of tuktuk hangyo, or to knock and plead.

\section{References}

Abe-Kim, J., Gong, F., \& Takeuchi, D. (2004). Religiosity, spirituality, and help-seeking among Filipino Americans: Religious clergy or mental health professionals? Journal of Community Psychology, 32, 675-689.

Amodeo, M., Lungren, L., Cohen, D., Chassler, D., \& D’ippolito, B. (2011). Barriers to implementing evidence-based practices in addiction treatment programs: Comparing staff reports on motivational interviewing, adolescent community reinforcement approach, assertive community treatment and cognitive behavioral therapy. Evaluation and Program Planning, 34, 382-389.

Baker, S., Daley, D.C., Donovan, D.M., \& Floyd, A.S. (2009). Stimulant abusers group to engage in 12-step programs. National Institute of Drug Abuse (NIDA-CTN-0031). Retrieved from http://ctndisseminationlibrary.org/PDF/888.pdf

Beck, A.T. (1970). Cognitive therapy: Nature and relation to behavioral therapy. Behavior Therapy, 1, 184-200.

Cepeda, M. (2016, September 22). DDB wants law institutionalizing community-based drug treatment. Rappler. Retrieved from http://www.rappler.com/nation/147020-ddblaw-institutionalize-community-based-drug-rehabilitation

Dangerous Drug Board. (2016). Community based treatment and rehabilitation resources. Retrieved from http://www.ddb.gov.ph/sidebar/301-community-basedtreatment-and-rehabilitation-resources.

Dueck, A., \& Byron, K. (2012). Community, spiritual traditions, and disasters in Chinese society. Pastoral Psychology, 61, 9931006. http://doi.org/10.1007/s11089-012-0437-0.

Ellis, A. (2001). Stress counseling: A rational emotive behavioral therapy approach. London: Sage Publications.

Enriquez, V. (1978). Kapwa: A core concept in Filipino social psychology. Philippine Social Sciences and Humanities Review, 42(1-4), 100-108.

Enriquez, V.G. (1992). From colonial to liberation psychology: The Philippine experience. Diliman, Quezon City, Phillipines: University of the Philippines Press.

Gilbert, P. (2014). The origin and nature of compassion focused therapy. British Journal of Clinical Psychology, 53, 6-41.

Haque, A. (2010). Mental health concepts in Southeast Asia: Diagnostic considerations and treatment implications. Psychology, Health and Medicine, 15, 127-134. http://doi.org/10.1080/13548501003615266

Hechanova, M.R., Tuliao, A.P., Teh, L.A., Alianan, A.S., \& Acosta, A. (2013). Problem severity, technology adoption, and intent to seek online counseling among overseas Filipino workers. Cyberpsychology Behavior Social Networking, 16, 613-617. http://doi.org/10.1089/cyber.2012.0648

Hechanova, M.R., Waelde, L.C., Docena, P., Alampay, L.P., Alianan, A., Flores, J., Ramos, P.A., \& Melgar, I. (2015). The development of Katatagan: A resilience intervention for Filipino disaster survivors. Philippine Journal of Psychology, 48, 105-131.

Hechanova, M.R., Waelde, L.C., \& Ramos, P.A. (2016). Evaluation of a group-based resilience program for Typhoon Haiyan survivors. Journal of Pacific Rim Psychology, 10, 1-10.

Hechanova, M.R., \& Waelde, L. (2017). The influence of culture on disaster mental health and psychosocial support interventions in Southeast Asia. Mental Health, Religion and Culture, $20,31-44$.

Hennig, R. (1983). Philippine values in perspective: An analytical framework. Philippine Sociological Review, 31, 55-64.

Human Rights Watch (2017). License to kill: Philippine police killings in Duterte's war against illicit drugs. Retrieved July 31, 2017, from https://www.hrw.org/report/2017/ 03/02/license-kill/philippine-police-killings-dutertes-warillicitdrugs.

Ilagan, J.R., Hechanova, M.R., Co, T., \& Pleyto, V. (2014). Bakit ka kumakayod? Developing a Filipino needs theory of motivation. Philippine Journal of Psychology, 47, 117-143

Ladrido-Ignacio, L. (2011). Basic framework: Transformation of victims of disasters to survivors. In L. Ignacio (Ed). Ginhawa (pp. 120-156). Quezon City, Philippines: Flipside Digital Content Company.

Lynch, F. (1962). Philippine values II: Social acceptance. Philippine Studies, 10, 82-99.

Ma, Y., Du, C., Cai, T., Han, Q., Yuan, H., Luo, T., ... Zhang, C. (2016). Barriers to community-based drug treatment: Implications for police roles, collaborations and performance indicators. Journal of International AIDS Society, 19, 64-70. 
McCarty, D., Braude, L., Lyman, D.R., Dougherty, R.H., Daniels, A.S., Ghose, S.S., \& Delphin-Rittmon, M.E. (2014). Substance abuse intensive outpatient: Assessing the evidence. Psychiatric Services, 65, 718-726.

McKleroy, V.S., Galbraith, J.S., Cummings, B., Jones, P., Harshbarger, C., Collins, C., ... ADAPT Team. (2006). Adapting evidence-based behavioral interventions for new settings and target populations. AIDS Education and Prevention, 18, 59-73.

Magill, M., \& Ray, L. (2009). Cognitive behavioural treatment with adult alcohol and illicit drug users: A meta-analysis of randomized clinical trials. Journal of Studies on Alcohol and Drugs, 70(4), 516-527.

Meyers, R.J., Roozen, H.G., \& Smith, J.E. (2011). The community reinforcement approach: An update of the evidence. Alcohol Research and Health, 33, 380-388.

Miller, W.R. \& Rollnick, S. (2012). Motivational interviewing: Preparing people for change. New York, NY: Guilford Press.

Mogato, M., \& Baldwin, C. (2017). Special report: Police describe kill rewards, staged crime scenes in Duterte's drug war. Reuters. Retrieved July 22, 2017, from http://www. reuters.com/article/us-philippines-duterte-policespecialrep-idUSKBN17K1F4

Nadal, K. (2011). Filipino American psychology: A handbook of theory, research, and clinical practice. Chichester, UK: John Wiley \& Sons.

Nakonz, J., \& Shik, A.W.Y. (2009). And all your problems are gone: Religious coping strategies among Philippine migrant workers in Hong Kong. Mental Health, Religion \& Culture, 12, 25-38. http://doi.org/10.1080/13674670802105252

Nelson, T.D., Steele, R.G., \& Mize, J.A. (2006). Practitioner attitudes towards evidence-based practice: Themes and challenges. Administrative Policy in Mental Health and Mental Health Services, 33, 398-409.

Nicotera, A.M. (1993). The importance of communication in interpersonal relationships. In A.M. Nicotera \& Associates (Eds.), Interpersonal communication in friend and mate relationships (pp. 3-12). Retrieved from http://www.sunypress.edu/pdf/52642.pdf

Ouimette, P.C., Finney, J.W., \& Moos, R.H. (1997). Twelve-step and cognitive behavioral treatment for substance abuse: A comparison of treatment effectiveness. Journal of Consulting and Clinical Psychology, 65, 230-240.

Palatino, M. (2017, January 9). Duterte's 'War on illicit drugs' in the Philippines: By the numbers. The Diplomat. Retrieved from http://thediplomat.com/2017/ 01/dutertes-war-on-illicitdrugs-in-the-philippines-by-thenumbers/

Parr, R. (2015). The use of expressive arts in making sense of traumatic experiences. Philippine Journal of Psychology, 48, 133-159.

Philippine Drug Enforcement Agency (PDEA). (2015). PDEA Annual Report. Retrieved from http://pdea.gov.ph/ our-accomplishments/annual-reports

Prochaska, J.O., DiClemente, C.C., \& Norcross, J.C. (1992). In search of how people change: Applications to the addictive behaviors. American Psychologist, 47, 1102-1114.
Raymundo, P.T. (2017) PNP resumes tokhang to look into $1.18 \mathrm{M}$ drug surrenderees. Retrieved from http://www.canadianinquirer.net/2017/03/11/pnp-resumestokhang-to-look-into-1-18-m-drug-surrenderees/

Reyes, J. (2015). Loob and kapwa: An introduction to a Filipino virtue ethics. Asian Philosophy, 26, 148-171.

Roozen, H.G., Boulogne, J.J., van Tulder, M.W., van den Brink, W., De Jong, C.A.J., \& Kerkhof, A.J.F.M. (2004). A systematic review of the effectiveness of the community reinforcement approach in alcohol, cocaine and opioid addiction. Drug and Alcohol Dependence, 74, 1-13. doi:10.1016/j.drugalcdep.2003.12.006

Sayegh, C., Huey, S., Zara, E., \& Jhaveri, K. (2017). Follow up treatment effects of contingency management and motivational interviewing on susbtance use: A meta-analysis. Psychology of Addictive Behaviors, 31, 403-414.

Substance Abuse Mental Health Services Administration (SAMHSA). (2006). Counselor's treatment manual: Matrix intensive outpatient treatment for people with stimulant use disorders (DHHS Publication No. SMA07-4152). Rockville, MD: Substance Abuse and Mental Health Services Administration.

Substance Abuse Mental Health Services Administration (SAMHSA). (2016). The eight dimensions of wellness. Retrieved from https://www.samhsa.gov/ wellness-initiative/eight-dimensions-wellness

Tuliao, A.P. (2014). Mental health help seeking among Filipinos: A review of the literature. Asia Pacific Journal of Counseling and Psychotherapy, 5, 124-136.

Tuliao, A.P., Velasquez, P.A.E., Bello, A.M., \& Pinson, M.J.T. (2016). Intent to seek counseling among Filipinos: Examining loss of face and gender. The Counseling Psychologist, 44, 353-382.

United Nations Office of Drug and Crime (UNODC). (2014). Community-based treatment and care for drug use and dependence. Retrieved from http://www.unodc.org/documents/southeastasiaandpacific/ cbtx/cbtx_brief_EN.pdf

United Nations Office of Drug and Crime (UNODC). (2015). Trainer's manual: Community-based services for people who use illicit drugs in Southeast Asia. Bangkok: United Nations Office for Drug and Crime for Southeast Asia and the Pacific.

Vuong, T., Nguyen, N, Le, G., Shanahan, M., Ali, R., \& Ritter, A. (2017). The political and scientific challenges in evaluating compulsory drug treatment centers in Southeast Asia. Harm Reduction Journal, 14, 1-14.

Windsor, L.C., Jemal, A., \& Alessi, E.J. (2015). Cognitive behavioral therapy: A meta-analysis of race and substance use outcomes. Cultural Diversity and Ethnic Minority Psychology, $21,300-313$.

World Health Organization (WHO). (2004). The ICD-10 symptom checklist. Retrieved from http://www.who.int/ substance_abuse/research_tools/en/english_icd10.pdf

World Health Organization (WHO). (2010). Brief assist: The ASSIST-linked brief intervention for hazardous and harmful substance use. Geneva, World Health Organization. Retrieved from http: apps.who.int 